\title{
Dissipative solitons with extreme spikes: bifurcation diagrams in the anomalous dispersion regime
}

\author{
Jose M. Soto-Crespo ${ }^{1}$, N. Devine ${ }^{2}$, and N. Akhmediev ${ }^{2}$ \\ ${ }^{1}$ Instituto de Óptica, IO-CSIC, Serrano 121, 28006 Madrid, Spain \\ ${ }^{2}$ Optical Sciences Group, Research School of Physics and Engineering, \\ The Australian National University, ACTON, ACT 2601, Australia
}

Compiled May 9, 2017

\begin{abstract}
Dissipative solitons with extreme spikes (DSES), previously thought to be rare solutions of the complex cubic-quintic Ginzburg-Landau equation, occupy in fact a significant region in its parameter space. The variation of any of its five parameters results in a rich structure of bifurcations. We have constructed several bifurcation diagrams that reveal periodic and chaotic dynamics of DSES. There are various routes to chaotic behavior of DSES including a sequence of period doubling bifurcations. It is well known that the complex cubic-quintic Ginzburg-Landau equation can serve as a master equation for the description of passively mode-locked lasers. Our results may lead to the observation of DSES in laser systems. (C) 2017 Optical Society of America
\end{abstract}

OCIS codes: $060.5530,140.7090,320.7110$

\section{Introduction}

There is recently a great interest in the study of extreme pulses in optics [1-4]. These are pulses with unexpectedly high amplitude which normally appear in a chaotic wave field. The study of extreme optical pulses may help to understand the phenomenon of extremes as a general concept that could be applied to other fields in physics including rogue waves in the oceans [5-7]. The latter are usually associated with dangers that ocean waves may represent. In contrast, extreme pulses in optics are safe to operate with. Moreover, they can be useful in applications providing ways for increasing the amplitude and the energy of the pulses. However, in order to do this, a better understanding of extreme pulses should be reached.

Extreme pulses were first observed in optical fibers as individual structures of supercontinuum generation [1]. Further research produced evidence that they are highamplitude solitons generated within a chaotic wave field $[2,3]$. Extreme optical pulses can alternatively be generated in wide aperture optical cavities [8-10] or in passively mode-locked lasers [11-13]. In the latter case, these high-amplitude pulses may appear in a chaotic wave field generated by the laser or they can be produced periodically on a regular basis. Transitions between the two regimes can be controlled by changing the parameters of the system. These transformations are complicated and represent a research area that is presently less studied. They have to be modeled theoretically or numerically in order to be better understood.

One of the most efficient techniques in modeling lasers with passive mode-locking is the master equation approach [14]. Master equation normally takes one or another form of the complex cubic-quintic GinzburgLandau equation $[15,16]$. This approach presents some advantages in finding new solutions with unusual properties, such as exploding and pulsating solitons [17], creeping solitons [18], chaotic solitons [19], dissipative soliton pairs [20], dissipative rogue waves [11,12], dissipative soliton resonances [21], and, more recently, spiny solitons [22]. Many of these predictions have been later observed experimentally [23-32], thus proving the fruitfulness of the approach.

An interesting effect recently discovered in numerical simulations is the existence of dissipative solitons with extreme spikes (DSESs) [33]. A DSES is a dissipative soliton with a low average amplitude that serves as a source of spikes emerging on top of it, either periodically [34] or chaotically [33]. Which of the operating regimes dominates, depends strongly on the parameters used in the CGLE. So far, DSESs have been found in two regions of parameters well separated from each other [33,34] suggesting that the phenomenon is not something exceptional but rather a common type of CGLE solutions. If this is the case, DSESs need certainly, further theoretical and experimental studies. Experimental studies can be difficult because high-resolution measurements should be done to resolve the narrow spikes of these solutions. In principle, the new solutions can be related to noiselike pulses as it has been conjectured in [22]. Noise-like pulses have been studied extensively [35-41] and they can serve as a bridge connecting theoretical and experimental studies on the subject of DSESs.

In this work, we continue the theoretical study on DSESs. Namely, we have found a wide parameter range of the CGLE with such solutions. This region of existence is large enough to claim that they are one of the generic solutions of the CGLE rather than exceptional formations. Moreover, we have found that they can show a large variety of dynamical behaviors. We have constructed bifurcation diagrams showing some specific 
transformations between DSES of different types. These diagrams could be useful in further progress on the subject both theoretical and experimental.

\section{Model}

As in previous works $[22,33,34,42,43]$, the specific model we are dealing with is the cubic-quintic complex Ginzburg-Landau equation:

$$
\begin{aligned}
i \psi_{z}+\frac{D}{2} \psi_{t t} & +|\psi|^{2} \psi+\nu|\psi|^{4} \psi \\
& =i \delta \psi+i \epsilon|\psi|^{2} \psi+i \beta \psi_{t t}+i \mu|\psi|^{4} \psi
\end{aligned}
$$

For passively mode-locked lasers, $t$ is the normalized time in a frame of reference moving with the group velocity, $\psi$ is the complex envelope of the optical field and $z$ is the propagation distance along the unfolded cavity. The meaning of the equation parameters on the left hand side is the following: $D$ denotes the cavity dispersion, being anomalous when $D>0$ and normal if $D<0$, and $\nu$ is the quintic refractive index coefficient. The coefficients of the dissipative terms that are written on the right-hand-side of Eq.(1) are the following: $\delta$ denotes linear gain/loss, $\beta$ is the gain bandwidth coefficient, $\epsilon$ and $\mu$ are the cubic and quintic gain/loss coefficients, respectively.

Several types of periodic solutions to this equation with unusual behaviors have been obtained in the anomalous dispersion regime [17]. These solutions experience periodic changes mainly in their width, while keeping almost constant their peak amplitude. In contrast, the solutions found here change their peak amplitude rather than width. These amplitude variations are extreme in the sense that the peak amplitudes exceed the average amplitude several times. In fact, this specific type of evolution with sharp spikes requires a special care in numerical procedures.

To solve Eq.(1) we have used a split-step technique with a fourth-order Runge-Kutta algorithm for solving the nonlinear part of the equation, while the linear part was solved in Fourier space. Modifications of this technique [44] have also been used. We used step sizes small enough to be sure that the parts of the solution with sharply growing amplitudes are finely discretized along both $z$ and $t$ axes.

In order to characterize DSESs, we use two pulse parameters: the energy, $Q(z)$,

$$
Q(z)=\int_{-\infty}^{\infty}|\psi(z, t)|^{2} d t
$$

and the peak amplitude,

$$
|\psi|_{\text {peak }}(z)=\max |\psi(z, t)|, \forall t .
$$

These two functions depend only on $z$. For pulsating solutions, maxima and minima of $Q$ will be denoted by $Q_{M}$ and $Q_{m}$ respectively.

\section{Bifurcation diagrams in $\epsilon$ and $\nu$}

The first difficulty in the searching of DSES solutions is finding an initial point in the parameter space supporting this type of solutions. This process may take weeks or months of searching and analyzing outputs. Once the first point is found, extending the region of parameters can be done in a systematic way, just shifting each parameter step by step around this point. Acting this way, we have found that the DSES solutions occupy a significant region in the space of parameters of the CGLE. We can vary any of the six parameters $\mu, \nu, D$, $\beta, \epsilon$ and $\delta$ in relatively wide intervals and the solutions still have the distinctive features of DSES. It is unrealistic to construct the whole region of existence in the six-dimensional space. As the parameters $\beta$ and $D$ are the only coefficients containing time derivatives, a simple time-rescaling may be used to eliminate one of them. Thus, effectively, we have to deal with five continuous real parameters, $\beta, \epsilon, \nu, \mu, \delta$, while $D$ can be restricted to be -1 or +1 . Nevertheless, even a five parameter space is too large to deal with. In this paper, we are concentrated on the anomalous dispersion regime, thus, fixing $D=+1$.
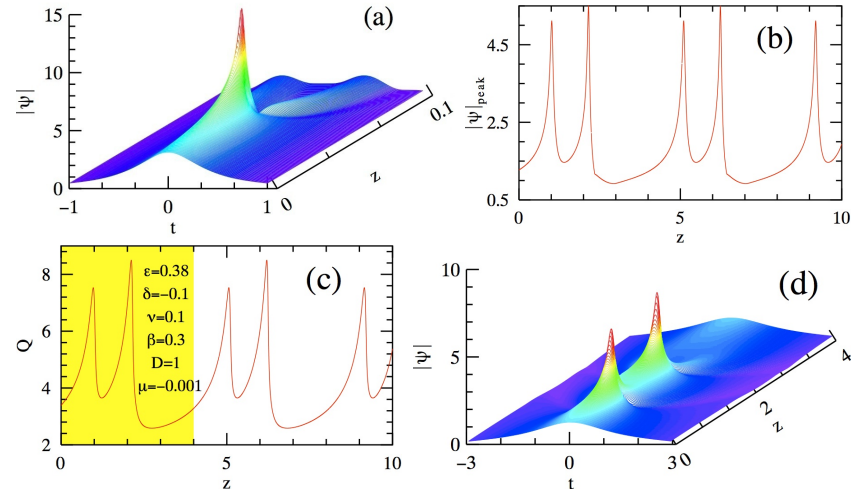

Fig. 1. (a) Pulsating dissipative soliton with one extreme spike per period for $\epsilon=1$. Other parameters are the same as given in the panel (c). Only one period of evolution is shown. (b) Double peak structure of the pulsating DSES obtained for $\epsilon=0.38$. (c) Evolution of the energy, Q, for the same case. (d) 3-D plot of the amplitude distribution of this solution for one period of evolution that is highlighted in yellow in (c).

Here, we illustrate the enormity of the size of the area of existence of DSESs, by changing all five parameters, namely $\epsilon, \nu, \beta \delta$ and $\mu$, while keeping $D=1$. Each of these parameters can be changed in relatively wide intervals and the solutions stay in the form of DSESs. Of course, the particular shape of these DSESs changes with the change of the equation parameters. Moreover, their dynamics may change from periodic pulsations to other types of evolution including chaotic behavior. Qualitative changes take the form of bifurcations. There are many bifurcations in each interval that we considered. 
In the simplest case, a bifurcation is a transition from a stationary solution to a pulsating solution of DSES type. These pulsating solutions can in turn be transformed into more complicated DSES with several spikes per period or those with asymmetric profiles. Examples of such bifurcation diagrams are presented below.

To start with, let us consider pulsating DSES, where the spikes normally appear at the center of the background ('mother') soliton. A typical example of this type of periodic solution is shown in Fig.1(a). When changing parameters of the CGLE, each spike on top of the soliton may split into two spikes of different amplitude. The first spike commonly has smaller amplitude than the second one. The solution stays periodic but after the bifurcation point each period contains two spikes rather than one. An example of such periodic solution with two spikes is shown in Fig.1(b). The evolution of the energy $Q$ for this solution is shown in Fig.1(c) and the 3-D plot of the optical field distribution on the plane $(\mathrm{t}, \mathrm{z})$ is shown in Fig.1(d) for the $z$-interval highlighted in yellow in (c). The latter represents one period of pulsations.
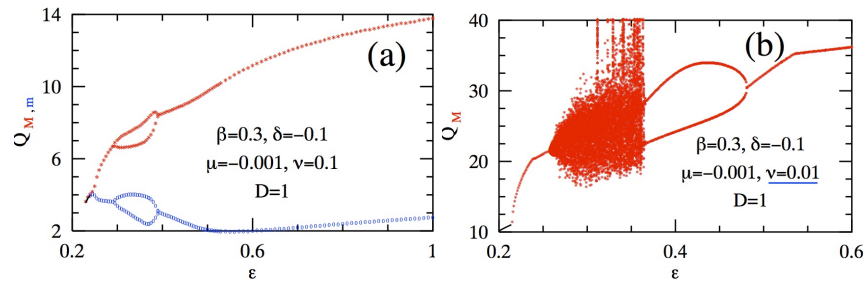

Fig. 2. Two bifurcation diagrams obtained when $\epsilon$ is varied. The difference between the two cases is the value of $\nu$. The plot in (a) shows the maxima and minima of the energy $Q$. It starts with $\epsilon=0.23$ where $Q_{M}=Q_{m}$. This corresponds to a stationary soliton without spikes. The first bifurcation at $\epsilon \approx 0.25$ leads to a pulsating soliton with a single spike per period. The bifurcation at $\epsilon \approx 0.3$ leads to a pulsating soliton with two spikes per period. These return back to pulsations with a single spike per period at $\epsilon \approx 0.4$. The second diagram (b) shows only maxima of $Q$. The inflection point at $\epsilon \approx 0.55$ corresponds to symmetry breaking. Below this point DSES solutions lose temporal symmetry and start to move. The bifurcation at $\epsilon \approx 0.48$ corresponds to a transition to solitons with two spikes per period. Further reduction of $\epsilon$ below $\approx 0.36$ switches to a chaotic dynamics. Nonmoving DSES with one spike per period are restored at $\epsilon \approx 0.25$. These are asymmetric solutions with the profile inverted after every period. An illustrative example of such behavior is shown in Fig.9(b) for other equation parameters. Below the inflection point, at $\epsilon=0.24$, the DSES fully recover their temporal symmetry. For $\epsilon$ less than 0.215 , the solution is transformed into a stationary soliton.

The above solutions have been obtained by varying the parameter $\epsilon$ in the CGLE. The corresponding bifurcation diagram is shown in Fig.2(a). The region $0.3 \leq \epsilon \leq 0.4$ corresponds to double spike generation per period. The splitting of the curves on the diagram demonstrates two different values of energy of the two spikes. The areas beyond this interval correspond to solutions having a single spike in each period. The upper (red) curve describes the maxima of energy while the lower (blue) curve corresponds to the minima. At the values of $\epsilon$ smaller than 0.23 , soliton solutions become unstable and vanish on propagation. In the interval $[0.23,0.25]$, maxima and minima of solitons merge into a single curve. This means that the pulsating DSES is transformed into a stationary soliton without spikes.

The double-peak DSES continues to exist when we change other parameters. For example, choosing $\epsilon=0.38$ in Fig.2(a) where the splitting is the largest, we can change, for instance, the parameter $\nu$. The corresponding bifurcation diagram is shown in Fig.3(a). Here, the spike splitting shows up in the interval of $\nu$ from $\nu \approx 0.08$ to $\nu=0.14$. Above this point, $\nu=0.14$, the soliton solution ceases to exist. Below the point $\nu \approx 0.08$, the solution turns back to a single spike solution per period. However, below the point $\nu \approx 0.035$, the double peak structure of DSES is restored. This region continues up to $\nu=0.0085$. Below this point, the DSESs are transformed into chaotic ones. They also move chaotically along the $t$-axis. This random motion is followed by the sequential splitting into several DSESs. As a result of this multiplication, the solution may occupy the whole numerical grid. This process is illustrated in Fig.3(b) calculated for $\nu=0.075$. For $\nu$ near zero, stationary dissipative solitons appear again. This is shown by the short black solid line in the upper left corner of Fig.3(a).
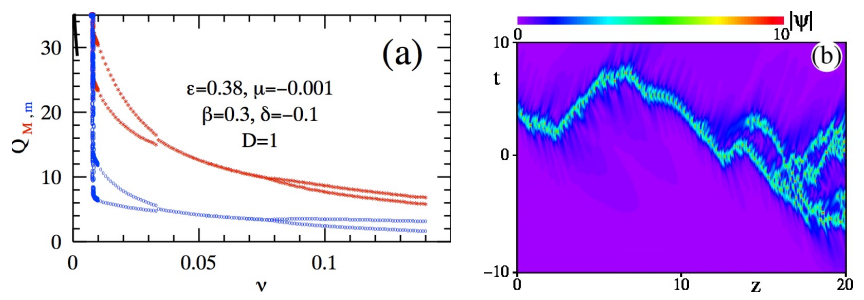

Fig. 3. (a) Soliton bifurcation diagram when changing the quintic nonlinear parameter $\nu$. Each of the two regions with split curves show two spikes per period. The left hand side region corresponds to moving DSES while the right hand side region corresponds to symmetric DSES with smaller variations of $Q$. The central region corresponds to non-moving DSES with a single spike per period. (b) Chaotic DSES evolution for $\nu=0.075$. Generation of additional DSES starts at $z \approx 14$.

The double spike solutions within the lower interval of $\nu$ are moving solitons. To give an example, the false color plot of DSES evolution at $\nu=0.01$ is shown in Fig.4(a). This plot shows explicitly the asymmetry and the motion of the soliton with spikes. In this particular case, the soliton moves to the left. However, equally well, it can move to the right if the initial conditions are 
mirror-imaged. The velocity of motion is fixed once the equation parameters are fixed. The solution has an asymmetric profile both in time and in spectral domains. The soliton is asymmetric in two ways. Firstly, the 'mother' soliton is asymmetric. Secondly, the location of the spikes is asymmetric relative to the 'mother' soliton. This can be seen clearly in Fig.4(a).

The evolution of the peak amplitude for this solution is shown in Fig.4(b) while the evolution of the energy is shown in Fig.4(c). The main difference from the case shown in Fig.1 is the higher modulation depth. Namely, the extreme amplitudes here are much higher than in Fig.1 and much higher than the amplitude of the 'mother' soliton.

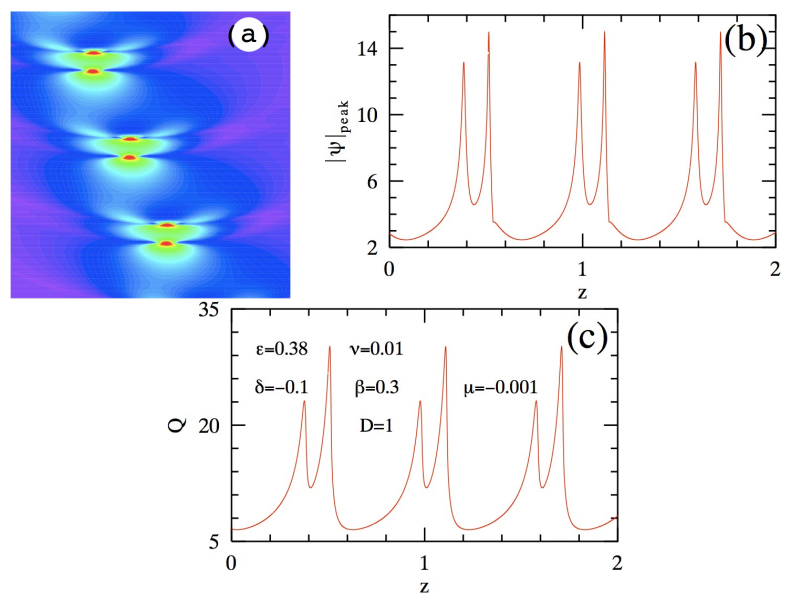

Fig. 4. An example of a moving DSES with two spikes per period. (a) False color plot of the amplitude on the $(t, z)$ plane. (b) Evolution of the peak amplitude. (c) Evolution of the energy $Q$. Parameters of the CGLE for this example are given in the panel (c).

When decreasing $\nu$ below 0.01 , the soliton evolution becomes chaotic. For example, when $\nu=0.008$, the spikes of random amplitude appear erratically at random values of $z$ along the soliton and may be shifted to the left or right across the soliton. When decreasing $\nu$ further to 0.0075, the solution splits into several DSESs randomly moving along the $t$-axis. One example of such evolution is shown in Fig.3(b). On further propagation, a multiplicity of randomly located interacting DSESs occupy the whole $t$-axis.

The bifurcation diagram in Fig.3(a) confirms that DSESs do exist in a wide range of parameters. Namely, we varied $\nu$ almost 20 times and DSESs do exist all along this range. We recall that $\nu$ is the coefficient in front of the quintic term and, in general, the solutions are very sensitive to its changes.

The curves on the bifurcation diagram may depend on the direction of the parameter change. When this happens, hysteresis loops can be observed. When such loops are present, they are usually small. Therefore, we did not concentrate on this aspects of the problem. The di- agram in Fig.3(a) is actually constructed starting from the point $\nu=0.1$ where two spikes per period exist. Other parts of the diagram are obtained when changing $\nu$ to the right or to the left from this point.

At the lowest point $\nu=0.01$, we fixed all parameters except $\epsilon$ and constructed the bifurcation diagram once again for changing $\epsilon$. This plot is shown in Fig.2(b). Only the upper curves for the maxima of energy are shown here. This diagram contains double spike DSES in the interval $0.37<\epsilon<0.48$. To the right from this interval, the DSES turn into single peak solution while to the left of the interval, we observe chaotic solutions with changing values of the peak amplitude from one period to the other. At $\epsilon \approx 0.26$ the chaotic solution turns into purely periodic one with a single spike per period. It moves in one direction. However, below the point $\epsilon=$ 0.25 , it moves back and forth periodically. Further below, after the inflection point at 0.24 , the solution becomes completely symmetric. It is transformed into a stationary solution at $\epsilon=0.215$. An important observation is that the interval of DSES existence here is also very large. It extends from $\epsilon=0.215$ to $\epsilon>0.6$. This is a very large interval for the cubic gain term.

\section{Bifurcation diagram in $\beta$}

As a next step in constructing bifurcation diagrams, we took the lowest point in Fig.3(a) with $\nu=0.01$ and varied $\beta$ keeping all other parameters fixed. The corresponding bifurcation diagram is shown in Fig.5(a). We have changed $\beta$ below the original point 0.3 up to 0.15 and above it up to 0.65 . This whole interval contains DSES solutions. For the sake of clarity and in order to keep high resolution along the vertical scale, in this diagram, only the maxima of energy are shown.
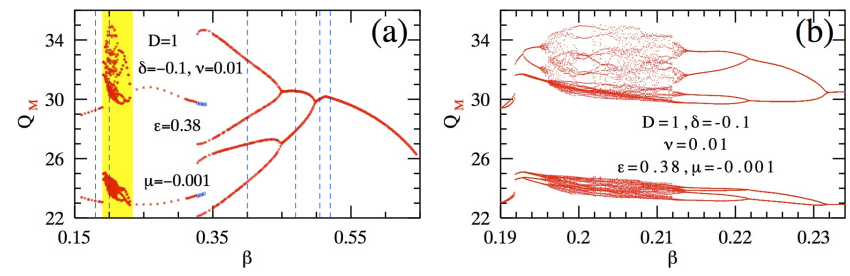

Fig. 5. (a) Bifurcation diagram with $\beta$ as the variable parameter. The part of the diagram highlighted in yellow is magnified in (b).

This diagram shows a rich bifurcation structure of solutions. The yellow region of (a) is further magnified in (b). This part of the diagram shows that the two main spikes experience a sequence of period doubling bifurcations before entering a region of chaotic dynamics. In order to reveal the nature of the solutions that correspond to each branch of the diagram, below we present one example for each branch. The points of $\beta$ that are chosen as representative of the type of solutions of the corresponding branch are shown by blue dashed vertical lines. A small region around $\beta=0.33$ shows up hysteresis 
with the corresponding bistability. The second solution in the bistability region is represented by blue points.

As explained above, for illustrative purposes, we have selected six particular DSES examples. They are shown in the twelve panels of Fig.6. The six panels on the left hand side show the evolution of the peak amplitude while the six panels on the right hand side show the evolution of the total energy. The case $\beta=0.18$ corresponds to a periodic solution with two spikes per period. The case $\beta=0.2$ is also a pulsating solution but the period of pulsations takes chaotic values. Thus, the solution is now chaotic but remains to be a single soliton.
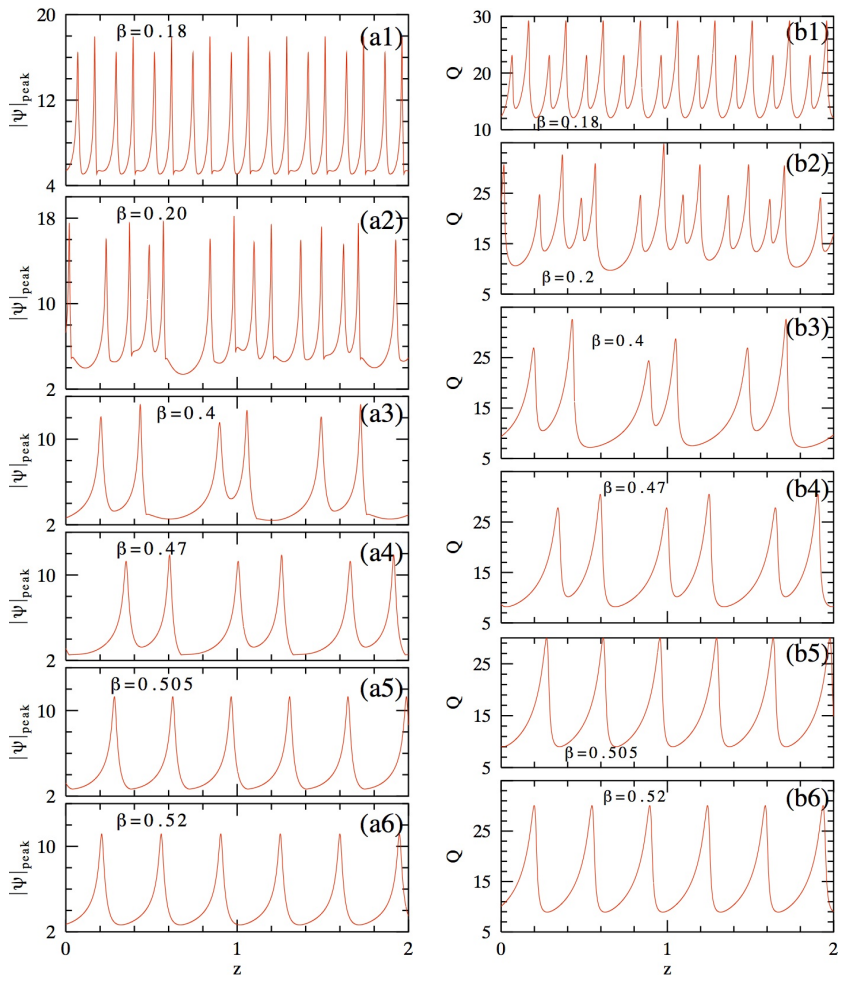

Fig. 6. Evolution of (a1-a6) the peak amplitude and (b1b6) the energy for the six cases marked in Fig.5 by blue dashed vertical lines. Parameter $\beta$ increases from top to bottom. Each panel on the left or on the right has a different vertical length but the same scale.

The case $\beta=0.4$ corresponds to a pulsating DSES with 4 extreme spikes per period. This is clear both from the bifurcation diagram in Fig. 5 and from the evolution curve in Fig.6. At $\beta=0.47$, after another bifurcation, the solution returns to a periodic one with two extreme spikes per period. Two other cases $\beta=0.505$ and $\beta=0.52$ correspond to pulsating solutions with one extreme peak per period. Although they look similar, these two solutions belong to different branches. This can be seen from the bifurcation curve in Fig.5. There is an inflection point on the curve located between the two cases at $\beta \approx 0.51$. It separates oscillating DSESs from symmetric ones with zero velocity. The period of oscillating solutions is twice the period of the curve $Q(z)$ seen in Fig.6. Similar inflection points can be seen on other bifurcation diagrams.

More detailed features of the DSES solutions can be seen from 3-D amplitude plots. Figure 7 shows the solution for the case $\beta=0.18$. This is a strictly periodic solution with two extreme spikes per period. The spikes are lined along two parallel straight lines. They are periodically shifted in transverse direction. The whole solution moves to the right keeping periodicity along the line of motion.
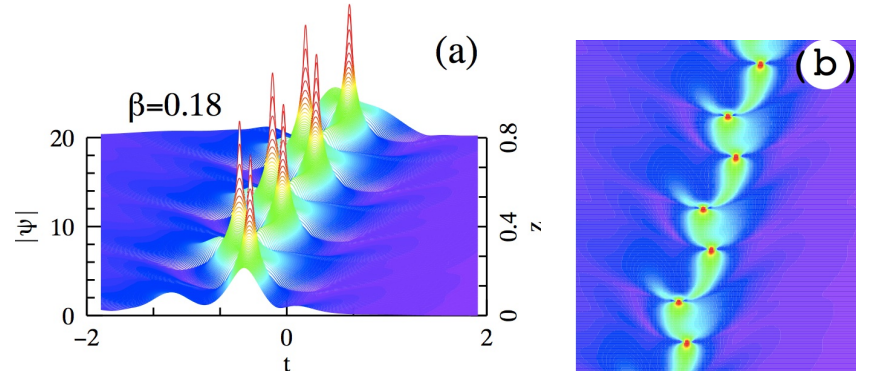

Fig. 7. (a) Field amplitude, $|\psi(z, t)|$, of the periodic DSES for $\beta=0.18$. Only $z$-values from 0 to 0.8 are shown. (b) Same as in (a) but in a false color format.

Two other examples of moving DSES for the cases $\beta=0.2$ and $\beta=0.4$ are shown in Fig.8. Fig.8(a) shows a chaotic moving solution while Fig.8(b) shows a period 2 moving solution. The first one has irregular periods of pulsation making it clear that it is a chaotic solution. Each period contains two extreme spikes per period just like in the case of Fig.7. One of them (precursor) is smaller and another one (successor) is bigger. In addition, there is a transverse shift of the soliton after each pulsation. As a result, they move with constantly changing velocity in (a) or fixed velocity in (b). The motion is always one way in the specific cases of Figs.8(a) and 8(b). However, other chaotic solutions may move with randomly changing direction and velocity.
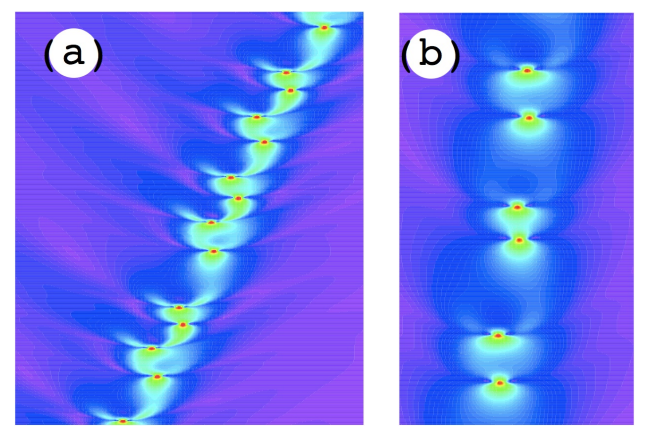

Fig. 8. Amplitude plots $(|\psi|)$ of DSES on the $(t, z)$ plane for (a) $\beta=0.2$ (chaotic moving solution), and (b) $\beta=0.4$ (period 2 moving solution). These correspond to the maximal amplitude plots in Figs.6(a2) and 6(a3) respectively. In each case, the spikes appear in pairs. 
There are many types of chaotic DSES. In order to see this more clearly, let us consider in more detail the region highlighted in yellow in Fig.5(a). A magnification of this region of the bifurcation diagram is shown in Fig.5(b). The most striking feature of this diagram is the multiplicity of bifurcations that takes place in a relatively narrow range of parameter change. This fact confirms that the CGLE has a myriad of different types of solutions. There are periodic and chaotic solutions in this region. The chaotic region can be entered through a sequence of period doubling bifurcations. This sequence can be seen in the region $0.216<\beta<0.231$. Other routes to chaos also exist at around $\beta=0.201$.

The chaotic behavior of dissipative solitons is known since the work [17]. Moreover, the transition from pulsating solitons to chaotic solitons through a sequence of period doubling bifurcations was found in [19]. This is a higher level generalization of the Feigenbaum phenomenon which has been discovered for the logistic map that is a representative system with one degree of freedom. The existence of similar sequence for partial differential equations with infinite number of degrees of freedom is not obvious. Moreover, the existence of such transition in the world of dissipative solitons is far from being trivial and this existence was revealed for the first time in [19].

In the present case, we made one step further in extending the principle. We have discovered a sequence of period doubling bifurcations for dissipative solitons with extreme spikes. The latter comprise a special subclass of the dissipative solitons that have been studied in [19]. Again, transition to chaotic dissipative solitons with extreme spikes from periodic ones is not a simple phenomenon. Our examples provide a starting point for these studies. We assume, many more discoveries can be done along this way.
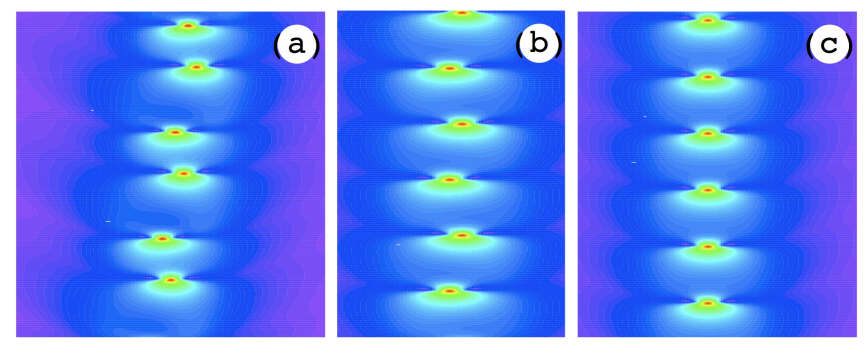

Fig. 9. Amplitude plots $(|\psi|)$ of DSES on the $(t, z)$-plane for (a) $\beta=0.47$, (b) $\beta=0.505$ and (c) $\beta=0.52$. In each case, $z$ changes (vertically) from 0 to 2 . There is a bifurcation point at $\beta \approx 0.51$. Below this point the solution moves (the cases (a) and (b)) while above it (the case (c)) the velocity is zero.

Three other DSES solutions for the cases $\beta=0.47$, $\beta=0.505$ and $\beta=0.52$ are shown in Fig.9. This region of parameter $\beta$ is characterized by regular periodic dynamics. All three cases show strict periodicity with period doubling in the case $\beta=0.47$. The DSES for $\beta=0.47$ oscillates and moves with positive velocity, the one for $\beta=0.505$ oscillates with a zero net velocity while the solution at $\beta=0.52$ is always centered and symmetric in $t$. There is a point $\beta \approx 0.51$ where zero velocity DSES loses stability. Instead, oscillating DSESs become stable. The inflection point that corresponds to this phenomenon is clearly seen on the bifurcation diagram shown in Fig.5(a).

\section{Bifurcation diagrams in $\delta$ and $\mu$}

For the sake of completeness, we also calculated bifurcation diagrams when either parameter $\delta$ or $\mu$ is changing. These two diagrams are shown in Figs.10(a) and 10(b) respectively. Other parameters are fixed. They are shown as inserts in these diagrams. Again, these diagrams demonstrate that DSESs exist in a wide range of $\delta$ and $\mu$ values confirming that the region of existence of DSESs in the five-dimensional parameter space is indeed exceptionally large.
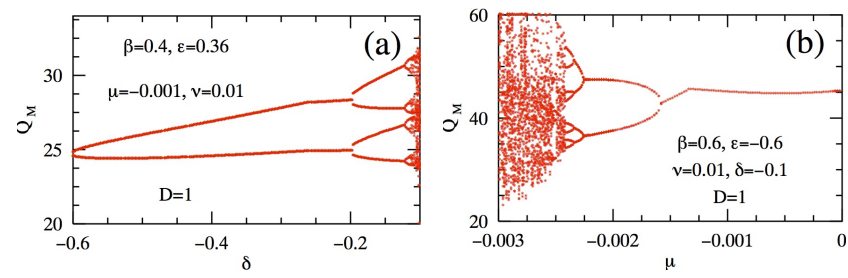

Fig. 10. Bifurcation diagrams when (a) $\delta$ changes, and (b) $\mu$ changes. Each of these diagrams is a clear case of a sequence of period doubling bifurcations leading to chaotic dynamics.

There are both periodic and chaotic DSES within the intervals shown in the diagrams. Each of the diagrams contains a region with a sequence of period doubling bifurcations, similar to those discussed above. Each of them leads to a region of chaotic solutions at $\delta \approx-0.11$ and $\mu \approx-0.0024$. These are also similar to the chaotic solutions shown in previous diagrams. Additionally, each of the diagrams contains the symmetry breaking point where zero velocity solutions are transformed into moving DSES. Otherwise, there are no new type of bifurcations in these diagrams that have not been discussed above.

\section{Conclusions}

The appearance of extreme spikes on top of wider dissipative solitons is a striking phenomenon that deserves further studies both theoretical and experimental. Here, we have shown that DSES occupy significant volumes of the parameter space of the complex cubic-quintic Ginzburg-Landau equation. Variation of any of the five parameters in this space results in a rich structure of bifurcations. We constructed several bifurcation diagrams that reveal periodic and chaotic dynamics of DSES. Pe- 
riodic solutions may have one or several pairs of spikes per period. They can be centered in $t$, oscillating around a fixed $t$, moving with a fixed or variable velocity, and even along a zigzag trajectory. Transition between them can take the form of bifurcations.

It is well known that the complex cubic-quintic Ginzburg-Landau equation can serve as a master equation for description of passively mode-locked lasers. Our new results may lead to observation of DSES in laser systems.

Individual sharp peaks could be difficult to detect with the existing equipment in optics. However, new techniques have been developed recently that may help in such measurements [45]. Namely, time stretching analogto-digital converters [46,47], time microscopes [48] and time lens magnifiers [49] may be able to resolve individual ultra-short pulses that could not be done with traditional pulse accumulating methods. In this sense, the theory and the experiment are progressing equally well and may allow us to be the witnesses of experimental observations of DSES soon.

\section{Acknowledgments}

The work of JMSC was supported by MINECO under contract TEC2015-71127-C2-1-R, and by C.A.M. under contract S2013/MIT-2790. N.A. and N.D. acknowledge the support of the Australian Research Council (DP150102057). All authors acknowledge the support of the Volkswagen Foundation.

\section{References}

1. D. R. Solli, C. Ropers, P. Koonath, and B. Jalali, Nature 450, 1054 (2007).

2. N. Akhmediev, J. M. Dudley, D. R. Solli and S. K. Turitsyn, Recent progress in investigating optical rogue waves, J. Opt., 15, 060201 (2013).

3. J. M. Dudley, F. Dias, M. Erkintalo and G. Genty, Instabilities, breathers and rogue waves in optics, Nature Photonics, 8, 755 - 764 (2014).

4. N. Akhmediev, B. Kibler, F. Baronio, M. Belić, W.P. Zhong, Y. Zhang, W. Chang, J. M. Soto-Crespo, P. Vouzas, P. Grelu, C. Lecaplain, K. Hammani, S. Rica, A. Picozzi, M. Tlidi, K. Panajotov, A. Mussot, A. Bendahmane, P. Szriftgiser, G. Genty, J. Dudley, A. Kudlinski, A. Demircan, U. Morgner, S. Amiraranashvili, C. Bree, G. Steinmeyer, C. Masoller, N. G. R. Broderick, A. F. J. Runge, M. Erkintalo, S. Residori, U. Bortolozzo, F. T. Arecchi, S. Wabnitz, C. G. Tiofack, S. Coulibaly and M. Taki, Roadmap on optical rogue waves and extreme events, J. Opt. 18, 063001 (2016).

5. A. Osborne, Nonlinear Ocean Waves $\&$ the Inverse Scattering Transform (Academic Press, 2010).

6. C. Kharif, E. Pelinovsky, and A. Slunyaev, Rogue Waves in the Ocean (Springer, 2009).

7. P. Müller, C. Garrett and A. Osborne, Oceanography 18, 66 (2005).

8. A. Montina, U. Bortolozzo, S. Residori and F. T. Arecchi, Non-Gaussian statistics and extreme waves in a nonlinear optical cavity, Phys. Rev. Lett., 103. 173901 (2009).

9. S. Residori, Bortolozzo, A. Montina, F. Lenzini and F. T. Arecchi, Rogue waves in spatially extended optical systems, Fluctuat. Noise Lett., 11. 1240014 (2012).

10. M. Onorato, S. Residori, U. Bortolozzo, A. Montina, and F. Arecchi, Rogue waves and their generating mechanisms in different physical contexts, Phys. Rep., 528, 47 - 89 (2013).

11. J. M. Soto-Crespo, Ph. Grelu and N. Akhmediev, Dissipative rogue waves: Extreme pulses generated by passively mode-locked lasers, Phys. Rev. E 84, 016604 (2011).

12. A. Zaviyalov, O. Egorov, R. Iliew and F. Lederer, Rogue waves in mode-locked fiber lasers, Phys. Rev. A 85 , 013828 (2012).

13. M. G. Kovalsky, A. A. Hnilo, and J. R. Tredicce, Extreme events in the Ti: sapphire laser, Opt. Lett., 36, 4449 (2011).

14. H. A. Haus, Mode-locking of lasers, IEEE J. Selected Topics in Quant. Electron., 6, 1173 (2000).

15. J. D. Moores, On the Ginzburg-Landau laser modelocking model with fifth-order saturable absorber term, Opt. Commun.v 96, 65 (1993).

16. A. Komarov, H. Leblond, and F. Sanchez, Quintic complex Ginzburg-Landau model for ring fiber lasers, Phys. Rev. E 72, 025604(R) (2005).

17. J. M. Soto-Crespo, N. Akhmediev and A. Ankiewicz, Pulsating, creeping and erupting solitons in dissipative systems, Phys. Rev. Lett., 85, 2937 (2000).

18. W. Chang, A. Ankiewicz and N. Akhmediev, Creeping solitons of the complex Ginzburg-Landau equation with a low-dimensional dynamical system model, Phys. Lett. A 362, 31 (2007).

19. N. Akhmediev, J. M. Soto-Crespo, and G. Town, Pulsating solitons, chaotic solitons, period doubling, and pulse coexistence in mode-locked lasers: Complex GinzburgLandau equation approach, Phys. Rev. E, 63, 056602 (2001).

20. N. Akhmediev, A. Ankiewicz and J. M. Soto-Crespo, Stable soliton pairs in optical transmission lines and fiber lasers, J. Opt. Soc. Am. B 15515 - 523 (1998).

21. Ph. Grelu, W. Chang, A. Ankiewicz, J. M. Soto-Crespo, and N. Akhmediev. Dissipative soliton resonance as a guideline for high-energy pulse laser oscillators, J. Opt. Soc. Am. B 27, 2336 - 2341 (2010).

22. W. Chang, J. M. Soto-Crespo, P. Vouzas and N. Akhmediev, Spiny solitons and noise-like pulses, J. Opt. Soc. America, 32, 1377 (2015).

23. S. T. Cundiff, J. M. Soto-Crespo and N. Akhmediev, Experimental evidence for soliton explosions, Phys. Rev. Lett., 88, 073903 (2002).

24. F. Amrani, A. Haboucha, M. Salhi, H. Leblond, A. Komarov and F. Sanchez, Dissipative solitons compounds in a fiber laser: Analogy with the states of the matter, Appl. Phys. B 99, 107 (2010).

25. L. M. Zhao, A. C. Bartnik, Q. Q. Tai and F. W. Wise, Generation of 8-nJ pulses from a dissipative-soliton fiber laser with a nonlinear optical loop mirror, Phys. Rev. E, 381942 (2013).

26. C. Cuadrado-Laborde, I. Armas-Rivera, A. Carrascosa, E. A. Kuzin, G. Beltrán-Pérez, A. Díez, and M. V. 
Andrés, Instantaneous frequency measurement of dissipative soliton resonant light pulses, Opt. Lett., 41, 5704 (2016).

27. K. Kieu, W. H. Renninger, A. Chong, and F. W. Wise, Sub-100 fs pulses at watt-level powers from a dissipativesoliton fiber laser, Opt. Lett., 34, 593 (2009).

28. D. Mortag, D. Wandt, U. Morgner, D. Kracht and J. Neumann, Sub-80-fs pulses from an all-fiber-integrated dissipative-soliton laser at $1 \mu \mathrm{m}$, Opt. Express, 19, 546 (2011).

29. D. S. Kharenko, O. V. Shtyrina, I. A. Yarutkina, E. V. Podivilov, M. P. Fedoruk, and S. A. Babin, Highly chirped dissipative solitons as a one-parameter family of stable solutions of the cubic-quintic Ginzburg-Landau equation, J. Opt. Soc. Am. B 28, 2314 (2011).

30. B. Chichkov, K. Hausmann, D. Wandt, U. Morgner, J. Neumann, D. Kracht, High-power dissipative solitons from an all-normal dispersion erbium fiber oscillator, Opt. Lett., 16, 2807 (2010).

31. X. Liu, Numerical and experimental investigation of dissipative solitons in passively mode-locked fiber lasers with large net-normal-dispersion and high nonlinearity, Opt. Express, 17, 22401 (2009).

32. Ph. Grelu, F. Belhache, F. Gutty and J. M. SotoCrespo, Phase-locked soliton pairs in a stretched-pulse fiber laser, Opt. Lett., 27, 966 - 968 (2002).

33. W. Chang, J. M. Soto-Crespo, P. Vouzas and N. Akhmediev, Extreme amplitude spikes in a laser model described by the complex Ginzburg-Landau equation, Opt. Lett., 40, 2949 - 2952 (2015).

34. W. Chang, J. M. Soto-Crespo, P. Vouzas and N. Akhmediev, Extreme soliton pulsations in dissipative systems Phys. Rev. E 92, 022926 (2015).

35. M. Horowitz, Y. Barad, and Y. Silberberg, Noiselike pulses with a broadband spectrum generated from an erbium-doped fibre laser, Opt. Lett., 22, 799 (1997).

36. L. M. Zhao, D. Y. Tang, T. H. Cheng, H. Y. Tam, and C. Lu, $120 \mathrm{~nm}$ bandwidth noise-like pulse generation in an erbium-doped fiber laser, Opt. Commun., 281, 157 (2008).

37. A. F. J. Runge, C. Aguergaray, N. G. R. Broderick, and M. Erkintalo, Coherence and shot-to-shot spectral fluctuations in noise-like ultrafast fiber lasers, Opt. Lett., 38, 4327 (2013).

38. J. U. Kang, Broadband quasi-stationary pulses in modelocked fiber ring laser, Opt. Commun., 182, 433 (2000).

39. O. Pottiez, R. Grajales-Coutino, B. Ibarra-Escamilla, E. A. Kuzin, and J. C. Hernández-García, Adjustable noiselike pulses from a figure-eight fiber laser, Appl. Opt., 50, E24 (2011).

40. M. Horowitz and Y. Silberberg, Control of noiselike pulse generation in erbium-doped fiber lasers, IEEE Photonic. Tech. L., 10, 1389 (1998).

41. A. Boucon, B. Barviau, J. Fatome, C. Finot, T. Sylvestre, M. W. Lee, P. Grelu, and G. Millot, Noiselike pulses generated at high harmonics in a partiallymode-locked km-long Raman fiber laser, Appl. Phys. B 106, 283 (2012).

42. Ph. Grelu and N. Akhmediev, Dissipative solitons for mode-locked lasers, Nature Photonics, 6, 84 (2012).

43. A. I. Korytin, A. Yu. Kryachko and A. M. Sergeev, Dissipative solitons in the complex Ginzburg-Landau equa- tion for femtosecond lasers, Radiophys. Quant. Electron., 44, 428, (2001).

44. J. Hult, A fourth-order Runge-Kutta in the interaction picture method for simulating supercontinuum generation in optical fibers, J. Lightwave Technol., 25, 3770, (2007).

45. B. Jalali, D. Solli, K. Goda, K. Tsia \& C. Ropers, Realtime measurements, rare events and photon economics. Europ. Phys. J. - Special Topics, 185, 145 (2010).

46. A. Bhushan, F. Coppinger \& B. Jalali, Time-stretched analogue-to-digital conversion, Electron. Lett., 34, 1081 (1998).

47. F. Coppinger, A. Bhushan \& B. Jalali, Photonic time stretch and its application to analog-to-digital conversion, IEEE Trans. Microwave Theory and Techniques, 47, 1309 (1999).

48. P. Suret, R. El Koussaifi, A. Tikan, C. Evain, S. Randoux, C. Szwaj \& S. Bielawski, Single-shot observation of optical rogue waves in integrable turbulence using time microscopy, Nature Commun., 7, 13136 (2016).

49. M. Närhi, B. Wetzel, C. Billet, S. Toenger, T. Sylvestre, J.-M. Merolla, R. Morandotti, F. Dias, G. Genty \& J. M. Dudley, Real-time measurements of spontaneous breathers and rogue wave events in optical fibre modulation instability, Nature Commun., 7, 13675 (2016). 